Annals of International Medical and Dental Research

E-ISSN: 2395-2822 | P-ISSN: 2395-2814

Vol-8, Issue-2 | March-April 2022

DOI: 10.53339/aimdr.2022.8.2.28

Page no- 219-232 | Section- Research Article (Haematology)

\title{
Pattern of Tumor Lysis Syndrome in Patients with Acute Lymphoblastic Leukemia
}

\section{Tanjina Afrin'1*, Abdul Aziz², Salahuddin Shah ${ }^{3}$, A B M Yunus, Amin Lutful Kabir5, Md. Adnan Hasan Masud6, Kazi Md Kamrul Islam7, A.Q.M. Ashraful Haque ${ }^{8}$, Zannatun Nesa9}

${ }^{1}$ Consultant, Department of Haematology, Green view clinic, Dhaka, Bangladesh.

Email: blueafrin@gmail.com,

Orcid ID: 0000-0002-9451-0815

2Professor, Department of Haematology, Bangabandhu Sheikh Mujib Medical University, Dhaka, Bangladesh.

Email: azizfcps@yahoo.com,

Orcid ID: 0000-0002-0416-0285

${ }^{3}$ Chairman, Department of Haematology, Bangabandhu Sheikh Mujib Medical University, Dhaka, Bangladesh.

Email: shah.fcps@gmail.com,

Orcid ID: 0000-0001-8903-6672

4Professor, Department of Haematology, Bangabandhu Sheikh Mujib Medical University, Dhaka, Bangladesh.

Email: abmyunus@gmail.com,

Orcid ID: 0000-0001-9144-6152

5Associate Professor, Department of Haematology, Bangabandhu Sheikh Mujib Medical University, Dhaka, Bangladesh. Email: aminlutful@gmai.com,

Orcid ID: 0000-0002-4132-3952

${ }^{6}$ Consultant, Department of Haematology, Bangabandhu Sheikh Mujib Medical University, Dhaka, Bangladesh.

Email: dr.adnan.hasan@gmail.com,

Orcid ID: 0000-0002-7216-4552

${ }^{7}$ Medical Officer, Department of Haematology,

Bangabandhu Sheikh Mujib Medical

University, Dhaka, Bangladesh.

Email: kamrulbsmmu@gmail.com,

Orcid ID: 0000-0001-9298-0023

8Resident physician, Department of Medicine, Colonel Malek Medical College, Manikganj, Bangladesh. Email: draqmhq@gmail.com,

Orcid ID: 0000-0003-3213-4276

9Specialist, Department of Haematology and SCT, Evercare Hospital Dhaka, Bangladesh. Email: zannat.rmc49@gmail.com,

Orcid ID: 0000-0003-2569-8217.

*Corresponding author

\begin{abstract}
Background: Prevention and treatment of tumor lysis syndrome (TLS) depends on immediate recognition of patients at risk. Therefore, we conducted this study to determine the frequency and risk factors of TLS in patients with acute lymphoblastic leukemia (ALL). Objective: The aim of the study was to observe the frequency of Tumor lysis syndrome in patients with Acute Lymphoblastic Leukemia. Material \& Methods: This cross-sectional study was conducted at the department of Haematology in Bangabandhu Sheikh Mujib Medical University (BSMMU), over a period of 12 months following approval of this protocol. Total 50 patients admitted with ALL were included in this study after careful history taking, examination and appropriate investigations fulfilling inclusion and exclusion criteria, irrespective of their gender, race, ethnic group and age. Ethical issues were ensured properly. After briefing the aims and objectives and potential risk and benefits, written informed consent was taken from each subject. Interviews were done by investigator herself using separate case record form. After editing and encoding, data was analyzed by computer with the help of SPSS 24. Results: The mean age of patients was $21.24 \pm 13.83$ (SD) years with majority aged less than 20 years (54\%) and male gender $(62 \%)$. Prevalence of TLS was found to be $26 \%(n=13)$, wherein spontaneous onset $(n=8,61.54 \%)$ and lab TLS $(n=9,69.23 \%)$ was more frequent than therapy induced TLS $(n=5,38.46 \%)$ and clinical TLS $(n=4$, $30.77 \%$ ). The most common biochemical changes occurred within 3 days before chemotherapy and 7 days after initiation of chemotherapy among TLS patients was hyperuricemia (69.23 and $76.92 \%$ respectively) and hyperkalaemia (61.54 and $69.23 \%$ respectively) with significant differences compared to non-TLS patients ( $p$ value $<0.05$ ). Initial WBC count and serum LDH of all patients was $52.51 \pm 78.70 \times 109 / \mathrm{L}$ and $1591.53 \pm 2195.47 \mathrm{U} / \mathrm{L}$ respectively, wherein majority patients with TLS had significantly higher WBC count $\geq 50 \times 109 / \mathrm{L}$ (61.54\%) and serum $\mathrm{LDH} \geq 1000 \mathrm{U} / \mathrm{L}$ (92.31\%) compared to non-TLS patients (16.22 and $21.62 \%$ respectively, $\mathrm{p}$ value $<0.05)$. Multivariate analysis showed that serum LDH $\geq 1000 \mathrm{U} / \mathrm{L}$ was the significant independent predictors of developing TLS (OR=13.07, 95\% CI: 1.93-101.23). Conclusions: TLS was commonly found in patients with ALL, wherein spontaneous onset and lab TLS was more common than therapy induced TLS and clinical TLS. However, a large multicenter study is needed to corroborate these findings.
\end{abstract}


Annals of International Medical and Dental Research

E-ISSN: 2395-2822 | P-ISSN: 2395-2814

Vol-8, Issue-2 | March-April 2022

DOI: $10.53339 /$ aimdr.2022.8.2.28

Page no- 219-232 | Section- Research Article (Haematology)

Received: 07 November 2021

Revised: 12 January 2022

Accepted: 24 January 2022

Published: 18 February 2022

Keywords:- Tumor, Lysis Syndrome, Acute Lymphoblastic, Leukemia.

\section{INTRODUCTION}

Tumor lysis syndrome (TLS) is defined as a combination of metabolic and electrolyte abnormalities (hyperuricemia, hyperkalemia, hyperphosphatemia, and secondary hypocalcemia) that occurs in patients with cancer, usually after the initiation of cytotoxic treatment but also spontaneously and may lead to serious clinical complications, including acute kidney injury and cardiac arrest. The tumor lysis syndrome is the most common disease-related emergency encountered by physicians caring for children or adults with hematologic cancers. [1] TLS is most frequently associated with hematological malignancies such as AML and ALL as well as NHL particularly Burkitt lymphoma,[2] and require prompt recognition followed by aggressive management. [3] Identification of tumor- and patient-specific risk factors for TLS and early recognition of laboratory and clinical TLS based on established criteria are essential for preventing TLS.[4] In the current classification system of Cairo and Bishop the tumor lysis syndrome can be classified as laboratory or clinical.[5] Laboratory tumor lysis syndrome requires that two or more of the following metabolic abnormalities occur within 3 days before or up to 7 days after the initiation of therapy: hyperuricemia, hyperkalemia, hyperphosphatemia, and hypocalcemia. Laboratory tumor lysis syndrome along with an increased creatinine level, seizures, cardiac dysrhythmia, or death is called clinical Tumor lysis syndrome. A few refinements could improve this classification. [1] First, it should be stipulated that two or more metabolic abnormalities be present simultaneously, because some patients may present with one abnormality, but later another one may develop that is unrelated to the tumor lysis syndrome (e.g., hypocalcemia associated with sepsis). Second, in contrast to Cairo and Bishop's definition, a $25 \%$ change from baseline should not be considered a criterion, since such increases are rarely clinically important unless the value is already outside the normal range. Third, any symptomatic hypocalcemia should constitute clinical tumor lysis syndrome.[2] Previous studies focused primarily on identifying patients at increased risk of TLS for the purpose of selecting those who may benefit from increased laboratory monitoring or urate oxidase therapy.[6] It is essential to identify patients at risk of tumor lysis syndrome because this life threatening condition may occur rapidly and is preventable. However, standardized procedures for assessing risk have been lacking until now.[7] There is lack of relevant local data for comparison. The incidence of TLS varies based on the underlying malignancy and the definition of TLS. Most incidence data are from older, retrospective studies. A pan European retrospective chart review identified TLS in $3.4 \%, 5.2 \%$ and $6.1 \%$ patients with AML, ALL and NHL respectively.[8] Prevalence of TLS 
Annals of International Medical and Dental Research E-ISSN: 2395-2822 | P-ISSN: 2395-2814

Vol-8, Issue-2 | March-April 2022

DOI: 10.53339/aimdr.2022.8.2.28

Page no- 219-232 | Section- Research Article (Haematology)

varies among different malignancies and there is no age or sex predilection. The risk is influenced by a number of characteristics including the type of malignancy, tumor burden, serum lactate dehydrogenase (LDH) levels, degree of involvement of the bone marrow and sensitivity of Tumor to chemotherapy. Patients health status, beyond malignancy-related factors, can also influence the risk of TLS, including presence of hypotension, dehydration, acidic urine (because of the greater propensity of UA to crystalize at low $\mathrm{Ph}$ ), oliguria, pre-cancer nephropathy, and previous experience with nephrotoxic agents. Crystal induced tissue injury occurs in the tumor lysis syndrome when calcium phosphate, uric acid, and xanthine precipitate in renal tubules and cause inflammation and obstruction.[9] A high level of solutes, low solubility, slow urine flow, and high levels of co-crystallizing substances favor crystal formation and increase the severity of the tumor lysis syndrome.[10] An elevated lactate dehydrogenase level ( $>2$ times the upper limit of the normal range) and an elevated uric acid level at presentation are associated with an increased risk of the tumor lysis syndrome and can be used to help classify borderline cases into a suitable risk group. If it is difficult to distinguish between two categories, treat the patient as if he or she is in the higher-risk category.[1] Hyperkalemia remains the most dangerous component of the tumor lysis syndrome because it can cause sudden death due to cardiac dysrhythmia. Patients should limit potassium and phosphorus intake during the risk period for the tumor lysis syndrome.[1] The purpose of this study was to determine the frequency of TLS in patients with Acute Lymphoblastic
Leukemia (ALL). This might be helpful in early identification and prevention of this serious complication, which can occur in Acute Lymphoblastic Leukemia, in order to initiate appropriate prophylactic and therapeutic measures and prevent the life threatening complications.

\section{MATERIAL AND METHODS}

This observational cross sectional study was conducted by Department of Hematology, Bangabandhu Sheikh Mujib Medical University, Dhaka, Bangladesh October 2019 to September 2020. Newly diagnosed case of Acute Lymphoblastic Leukemia patients admitted in the department of Hematology in BSMMU, Dhaka during the study period. This study sampling method was consecutive sampling. Inclusion criteria were all newly diagnosed Acute Lymphoblastic Leukemia patients on the basis of $\mathrm{CBC}$ with PBF, bone marrow study and immunophenotyping. Exclusion criteria were relapsed and secondary case of Acute Lymphoblastic Leukemia, known case of epilepsy and other convulsive disorder and known case of chronic kidney disease. Tumor lysis syndrome (TLS) describes the metabolic derangements that occur with Tumor breakdown following the initiation of cytotoxic therapy. TLS results from the rapid destruction of malignant cells and the abrupt release of intracellular ions, nucleic acids, proteins and their metabolites into the extracellular space. These metabolites can overwhelm the body's normal homeostatic mechanisms and cause hyperuricemia, hyperkalemia, hyperphosphatemia, hypocalcaemia and uremia. This study was a hospital-based, cross sectional descriptive observational study and was conducted in the 
Annals of International Medical and Dental Research

E-ISSN: 2395-2822 | P-ISSN: 2395-2814

Vol-8, Issue-2 | March-April 2022

DOI: $10.53339 /$ aimdr.2022.8.2.28

Page no- 219-232 | Section- Research Article (Haematology)

Departments of Haematology, Bangabandhu Sheikh Mujib Medical University, Dhaka. The study was approved by the local ethical committee and all patients gave their informed consent to take part in this investigation.

The duration of the study was 12 months and included 73 number of Acute Lymphoblastic Leukemia patient who had attained in inpatient or out-patient department of Haematology in BSMMU meeting the inclusion \& exclusion criteria. The aim, benefit and the likely risk was explained to the patient if he/she agree then informed written consent was taken. Clinical history was taken and physical examination (Anemia, Dehydration, Lymphadenopathy, Purpuric rash, Organomegaly, seizure, oliguria, oedema) was evaluated. Baseline electrocardiography of each patient was recorded at presentation and was repeated if patient developed any symptoms or noticed to have arrhythmia. All the documents necessary to confirm the diagnosis were collected. Venous blood samples were drawn in EDTA tube for total leucocyte count (TLC) and were analyzed on Sysmex analyzer. Venous blood samples were drawn in lithium heparin coated vacutainers to determine the levels of serum phosphate, potassium, uric acid, creatinine, calcium, albumin and serum lactate dehydrogenase $(\mathrm{LDH})$ at presentation and then were checked daily from 3 days before and 7 days after initiation of chemotherapy. These samples were run on NOVA biomedical-4 automated analyzer for serum potassium and on Hitachi 911 automatic analyzer for serum uric acid, creatinine, calcium, phosphate, albumin and LDH. Qualitative variables (eg. sex) of this study were expressed as percentage.
Quantitative variables (eg. age) were expressed as mean \pm standard deviation. Categorical comparisons were performed using chi-square test. Numerical variables were presented as mean, median and standard deviation. Multiple logistic regression analysis was done to identify the best fitted determinants.

\section{RESULTS}

This observational cross-sectional study was performed in the department of Haematology, BSMMU, Dhaka over a period of one year. After careful history taking, examination and appropriate investigations fulfilling inclusion and exclusion criteria, total 50 patients admitted with acute Lymphoblastic Leukemia (ALL) were included in this study, irrespective of their gender, race, ethnic group and age. The main aim of the study was to observe the frequency of Tumor lysis syndrome (TLS) in patients with acute lymphoblastic leukemia. The results of the study were arranged in [Table and Figure]. Details of the study result are described below. The mean age of all patients was 21.24 \pm 13.83 years ranging from 370 years. Maximum (54\%) patients were aged less than 20 years. Greater part of the patients was male (62\%) compared to female (38\%). All patients of this study had complained about fever $(100 \%)$ followed by progressive pallor $(98 \%)$, history of blood transfusion (30\%), bleeding manifestation (26\%) and palpitation $(2 \%)$. The most common clinical finding was bony tenderness (58\%) followed in decreasing order splenomegaly (48\%), lymphadenopathy $(46 \%)$, oral ulcer $(46 \%)$, hepatomegaly $(36 \%)$, abdomen distension (10\%), oliguria (8\%), leg oedema $(8 \%)$, ascites $(4 \%)$, basal crepitation $(4 \%)$, jaundice $(2 \%)$, haematuria $(2 \%)$, and facial oedema (2\%). [Table 3] showed the 
Annals of International Medical and Dental Research E-ISSN: 2395-2822 | P-ISSN: 2395-2814

Vol-8, Issue-2 | March-April 2022

DOI: 10.53339/aimdr.2022.8.2.28

Page no- 219-232 | Section- Research Article (Haematology)

laboratory findings of study patients at the time of initial assessment wherein mean total WBC count, platelet count and serum LDH were higher than normal limit. Major part of the study patients had B-cell type ALL (66\%) whereas rest $34 \%$ had T-cell type. [Figure 4] showed that $13(26 \%)$ of the study population have tumor lysis syndrome. [Figure 6] showed that $8(61.54 \%)$ of ALL patients with tumor lysis syndrome had spontaneous onset whereas 5 patients (38.46\%) developed TLS after initiation of chemotherapy. Maximum patients with TLS had significantly higher number of hyperuricemia (69.23\%) and hyperkalaemia $(61.54 \%)$ and high serum creatinine level $(23.08 \%)$ compared to non-TLS patients (2.7, 27.03 and $0 \%$ respectively) within 3 days before chemotherapy. Besides, majority patients with TLS had significantly greater number of hyperuricemia (76.92\%), hyperkalaemia
(69.23\%) and hyperkalaemia (46.15\%) compared to non-TLS patients $(0,18.92$, and $0 \%$ respectively) within 7 days after initiation of chemotherapy. There had significant difference in distribution of immunophenotype, lymphadenopathy, hepato-splenomegaly, WBC and serum LDH level wherein majority patients from TLS group had T-cell type ALL (61.54\%), lymphadenopathy (92.31\%), hepatosplenomegaly (69.23\%), WBC $\geq 50 \quad x 109 / \mathrm{L}$ (61.54\% and serum LDH $\geq 1000 \mathrm{U} / \mathrm{L}$ (92.31\%). Multivariate logistic regression analysis to determine the independent predictors of developing TLS among ALL patients showed that serum $\mathrm{LDH} \geq 1000 \mathrm{U} / \mathrm{L}$ was the significant independent predictors of developing TLS $(\mathrm{OR}=13.07,95 \% \mathrm{CI}: 1.93-101.23)$ after adjusting for age, gender, lymphadenopathy, hepatosplenomegaly and WBC level.

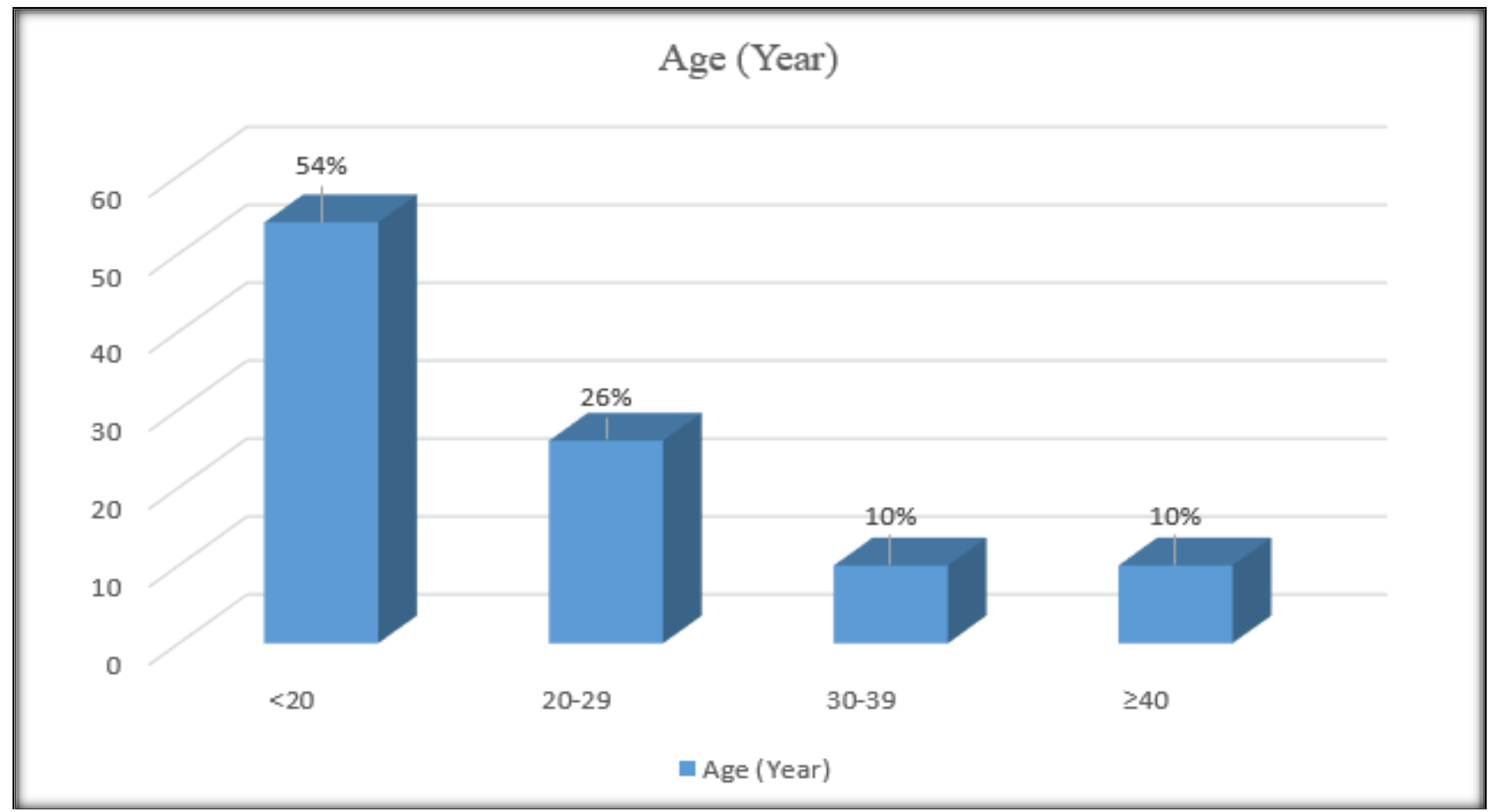

Figure 1: Age distribution of patients $(n=50)$ 


\section{Gender Distribution}

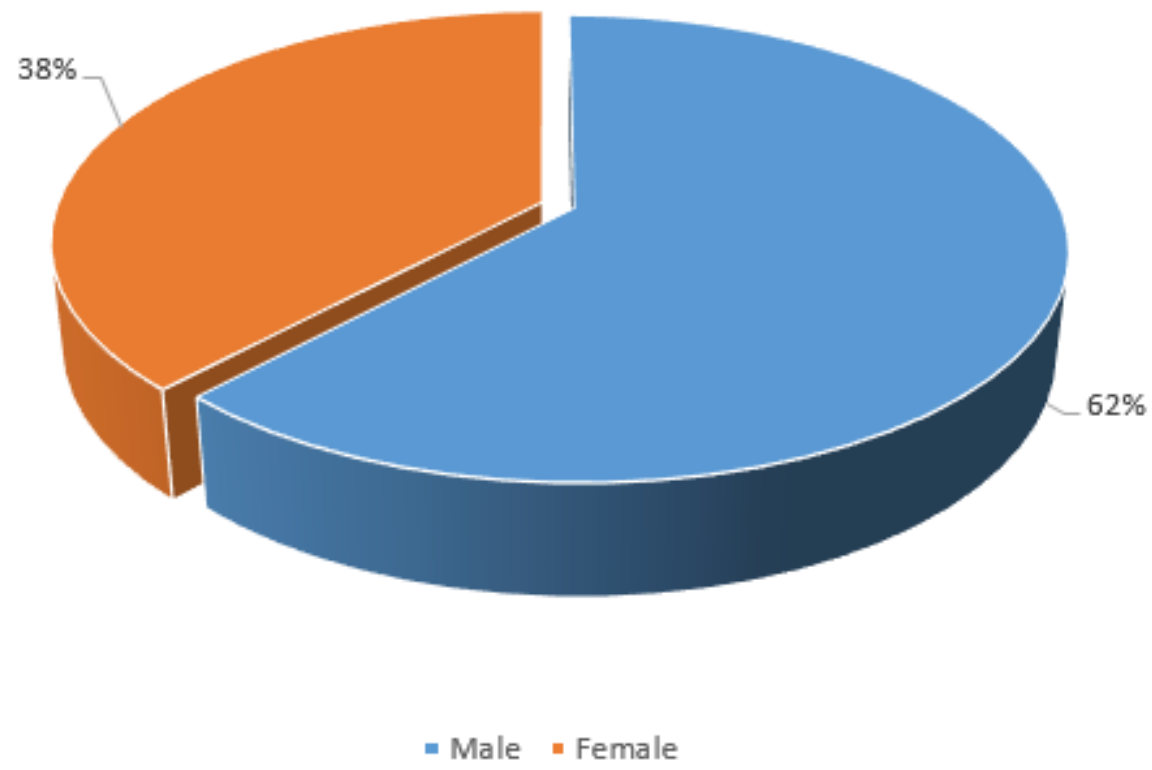

Figure 2: Gender distribution of patients $(n=50)$

Table 1: Chief complaints of study patients $(n=50)$

\begin{tabular}{|l|l|l|}
\hline Chief complaints & $\mathbf{n}$ & \% \\
\hline Fever & 50 & 100.0 \\
\hline Progressive pallor & 49 & 98.0 \\
\hline History of blood transfusion & 15 & 30.0 \\
\hline Bleeding manifestation & 13 & 26.0 \\
\hline Palpitation & 1 & 2.0 \\
\hline Convulsion & 0 & 0.0 \\
\hline
\end{tabular}

Table 2: Clinical findings of study patients $(n=50)$

\begin{tabular}{|l|l|l|}
\hline Clinical findings & $\mathbf{n}$ & $\%$ \\
\hline Bony tenderness & 29 & 58.0 \\
\hline Splenomegaly & 24 & 48.0 \\
\hline Lymphadenopathy & 23 & 46.0 \\
\hline Oral ulcer & 23 & 46.0 \\
\hline Hepatomegaly & 18 & 36.0 \\
\hline Distended abdomen & 5 & 10.0 \\
\hline
\end{tabular}


Annals of International Medical and Dental Research

E-ISSN: 2395-2822 | P-ISSN: 2395-2814

Vol-8, Issue-2 | March-April 2022

DOI: 10.53339/aimdr.2022.8.2.28

Page no- 219-232 | Section- Research Article (Haematology)

\begin{tabular}{|l|l|l|}
\hline Oliguria & 4 & 8.0 \\
\hline Leg oedema & 4 & 8.0 \\
\hline Ascitis & 2 & 4.0 \\
\hline Basal Crepitation & 2 & 4.0 \\
\hline Jaundice & 1 & 2.0 \\
\hline Haematuria & 1 & 2.0 \\
\hline Facial Oedema & 1 & 2.0 \\
\hline
\end{tabular}

Table 3: Laboratory findings of the study participants $(n=50)$

\begin{tabular}{|l|l|l|}
\hline Parameters & Range (minimum-maximum) & Mean \pm SD \\
\hline Total WBC count $(x 109 / \mathrm{L})$ & $6-435$ & $52.51 \pm 78.70$ \\
\hline $\mathrm{Hb} \%(\mathrm{gm} / \mathrm{dl})$ & $4.1-13.4$ & $9.25 \pm 1.49$ \\
\hline Platelet $(\mathrm{x} 109 / \mathrm{L})$ & $5-20000$ & $505.20 \pm 2815.29$ \\
\hline Blast in PBF $(\%)$ & $10-90$ & $53.80 \pm 21.251$ \\
\hline S. $\mathrm{LDH}(\mathrm{U} / \mathrm{L})$ & $198-11863$ & $1591.53 \pm 2195.47$ \\
\hline S. uric acid $(\mathrm{mg} / \mathrm{dL})$ & $1.40-30.03$ & $5.76 \pm 4.61$ \\
\hline S. inorganic phosphate $(\mathrm{mg} / \mathrm{dL})$ & $2.30-7.70$ & $4.19 \pm 1.20$ \\
\hline S. potassium (mmol/L) & $2.50-5.40$ & $3.92 \pm 0.56$ \\
\hline S. calcium $(\mathrm{mg} / \mathrm{dL})$ & $1.10-11.80$ & $8.33 \pm 2.08$ \\
\hline S. creatinine $(\mathrm{mg} / \mathrm{dL})$ & $0.19-3.59$ & $89 \pm 0.55$ \\
\hline
\end{tabular}

\section{Immunophenotype}

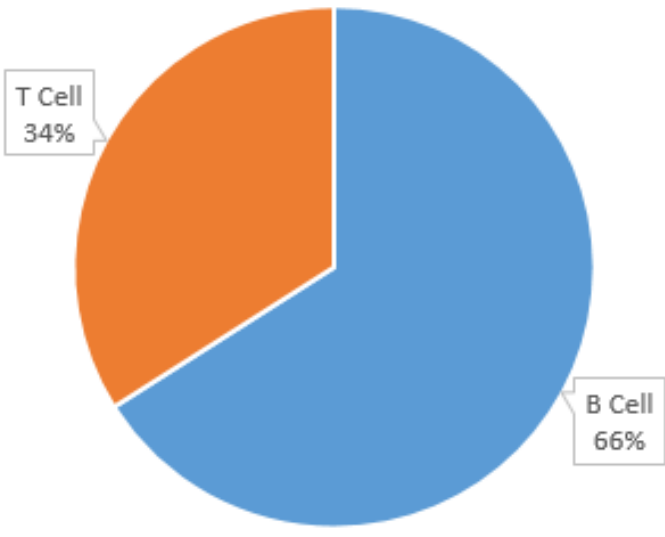

- BCell - TCell

Figure 3: Immunophenotyping of ALL patients $(n=50)$ 


\section{Tumor lysis syndrome}

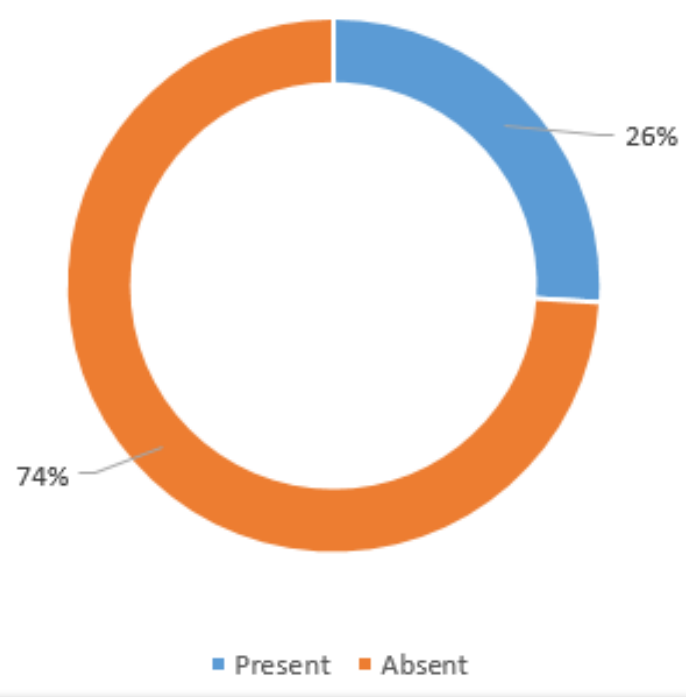

Figure 4: Prevalence of TLS among ALL patients $(n=50)$

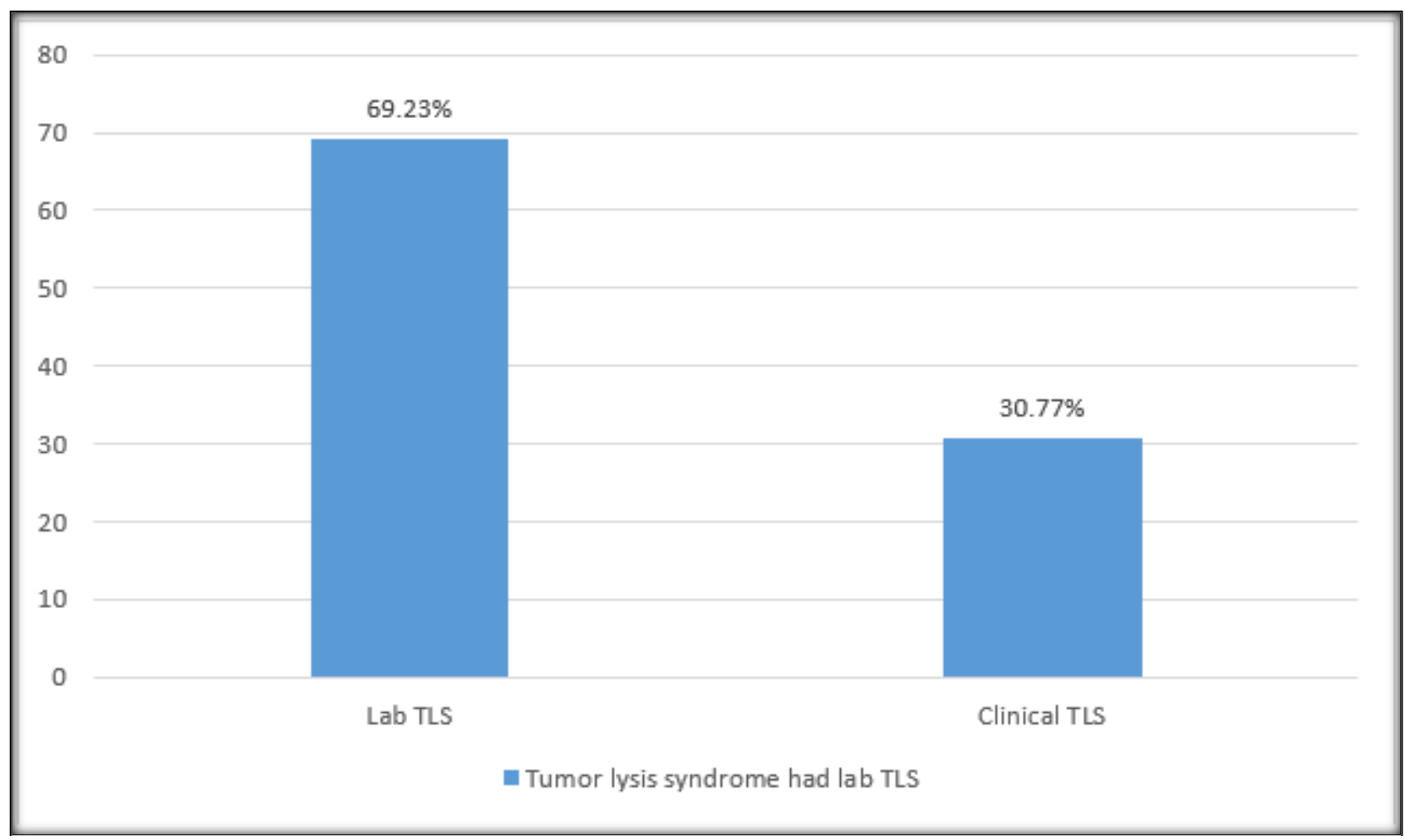

Figure 5: Distribution of lab and clinical TLS among TLS patients $(n=13)$ 


\section{TLS according to onset}

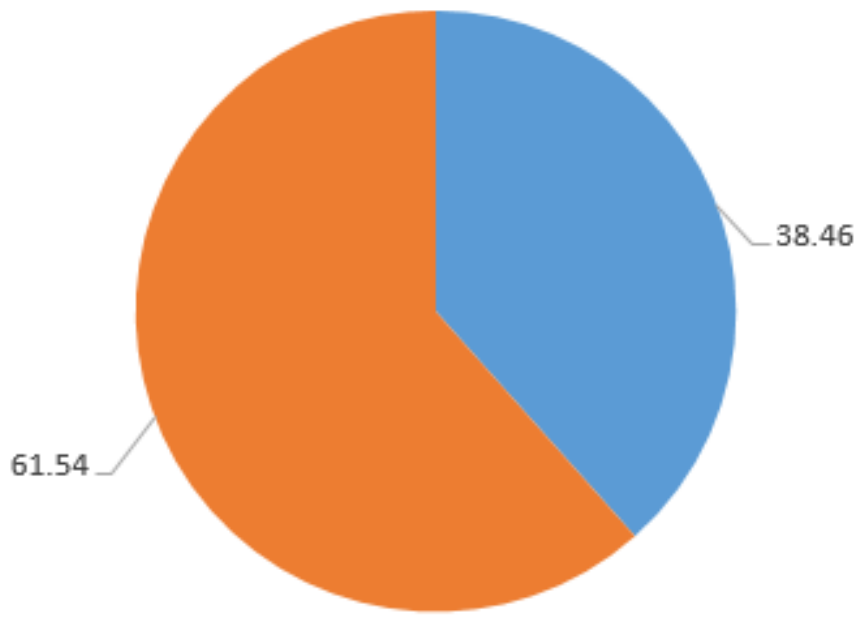

Epontaneous TLS $\quad$ Therapy related TLS

Figure 6: Distribution of TLS according to onset $(n=13)$

Table 4: Prevalence of biochemical abnormalities in patients with ALL $(n=50)$

\begin{tabular}{|c|c|c|c|c|c|}
\hline \multirow[t]{3}{*}{ Variables } & \multicolumn{4}{|c|}{ Tumor lysis syndrome } & \multirow[t]{3}{*}{$\mathrm{p}$ value } \\
\hline & \multicolumn{2}{|l|}{ Yes } & \multicolumn{2}{|l|}{ No } & \\
\hline & $\mathrm{n}=\mathbf{1 3}$ & $\%$ & $\mathrm{~N}=37$ & $\%$ & \\
\hline \multicolumn{6}{|c|}{ Within 3 days before chemotherapy } \\
\hline Hyperuricemia (>8.0 mg/dl) & 9 & 69.23 & 1 & 2.7 & $<0.001$ \\
\hline Hyperkalaemia $(>4.5 \mathrm{mg} / \mathrm{dl})$ & 8 & 61.54 & 10 & 27.03 & 0.043 \\
\hline Hyperkalaemia ( $\geq 6.0 \mathrm{mmol} / \mathrm{L})$ & 1 & 7.69 & 0 & 0.0 & 0.260 \\
\hline High s. creatinine (>1.8 mg/dl) & 3 & 23.08 & 0 & 0.0 & 0.015 \\
\hline Hypocalcaemia $(<7.0 \mathrm{mg} / \mathrm{dl})$ & 0 & 0.0 & 0 & 0.0 & - \\
\hline \multicolumn{6}{|c|}{ Within 7 days after initiation of induction chemotherapy } \\
\hline Hyperuricemia (>8.0 mg/dl) & 10 & 76.92 & 0 & 0.0 & $<0.001$ \\
\hline Hyperkalaemia (>4.5 mg/dl) & 9 & 69.23 & 7 & 18.92 & 0.002 \\
\hline Hyperkalaemia ( $\geq 6.0 \mathrm{mmol} / \mathrm{L})$ & 6 & 46.15 & 0 & 0.0 & $<0.001$ \\
\hline High s. creatinine $(>1.4 \mathrm{mg} / \mathrm{dl})$ & 2 & 15.38 & 0 & 0.0 & 0.064 \\
\hline Hypocalcaemia $(<7.0 \mathrm{mg} / \mathrm{dl})$ & 2 & 15.38 & 0 & 0.0 & 0.064 \\
\hline
\end{tabular}


Annals of International Medical and Dental Research E-ISSN: 2395-2822 | P-ISSN: 2395-2814

Vol-8, Issue-2 | March-April 2022

DOI: $10.53339 /$ aimdr.2022.8.2.28

Page no- 219-232 | Section- Research Article (Haematology)

Table 5: Association of different factors with tumor lysis syndrome $(n=50)$

\begin{tabular}{|c|c|c|c|c|c|}
\hline \multirow[t]{3}{*}{ Variables } & \multicolumn{4}{|c|}{ Tumor lysis syndrome } & \multirow[t]{3}{*}{ p value } \\
\hline & \multicolumn{2}{|c|}{ Yes } & \multicolumn{2}{|l|}{ No } & \\
\hline & $n=13$ & $\%$ & $\mathrm{n}=37$ & $\%$ & \\
\hline \multicolumn{6}{|l|}{ Age (in years) } \\
\hline$<20$ & 7 & 53.85 & 20 & 54.05 & \multirow[t]{2}{*}{1.00} \\
\hline$\geq 20$ & 6 & 46.15 & 17 & 45.95 & \\
\hline \multicolumn{6}{|l|}{ Gender } \\
\hline Male & 9 & 69.23 & 22 & 59.46 & \multirow[t]{2}{*}{0.742} \\
\hline Female & 4 & 30.77 & 15 & 40.54 & \\
\hline \multicolumn{6}{|l|}{ Immunophenotyping } \\
\hline B cell type & 5 & 38.46 & 28 & 75.46 & \multirow[t]{2}{*}{0.021} \\
\hline T cell type & 8 & 61.54 & 9 & 24.32 & \\
\hline \multicolumn{6}{|l|}{ Organomegaly } \\
\hline Lymphadenopathy & 12 & 92.31 & 11 & 29.73 & $<0.001$ \\
\hline Hepato-Splenomegaly & 9 & 69.23 & 8 & 21.62 & 0.005 \\
\hline \multicolumn{6}{|c|}{ Initial WBC count $(\times 109 / \mathrm{L})$} \\
\hline$<50$ & 5 & 38.46 & 31 & 83.78 & \multirow[t]{2}{*}{0.004} \\
\hline$\geq 50$ & 8 & 61.54 & 6 & 16.22 & \\
\hline \multicolumn{6}{|l|}{ S. LDH level (U/L) } \\
\hline$<1000$ & 1 & 7.69 & 29 & 78.38 & \multirow[t]{2}{*}{$<0.001$} \\
\hline$\geq 1000$ & 12 & 92.31 & 8 & 21.62 & \\
\hline
\end{tabular}

Table 6: Multivariate logistic regression to detect independent predictors of developing TLS among ALL patients $(n=50)$

\begin{tabular}{|l|l|l|l|l|}
\hline Predictor & \multirow{2}{*}{ OR } & $\mathbf{9 5 \% \text { CI }}$ & p value \\
\cline { 3 - 5 } & & Lower & Upper & \\
\hline Age $\geq 20$ years & 2.44 & 0.28 & 21.40 & 0.421 \\
\hline Male & 1.02 & 0.82 & 13.54 & 0.848 \\
\hline lymphadenopathy & 6.95 & 0.45 & 107.28 & 0.165 \\
\hline Hepato-splenomegaly & 3.17 & 0.29 & 34.75 & 0.344 \\
\hline T cell type & 3.95 & 0.42 & 36.81 & 0.228 \\
\hline Initial WBC count $(>50.0 \times 109 / \mathrm{L})$ & 2.12 & 0.91 & 7.16 & 0.714 \\
\hline S. LDH $(\geq 1000 \mathrm{U} / \mathrm{L})$ & 13.07 & 1.93 & 101.23 & 0.015 \\
\hline
\end{tabular}

\section{DISCUSSION}

Tumor lysis syndrome (TLS) is a lifethreatening oncological emergency with high morbidity and mortality in patients with acute lymphoblastic leukaemia (ALL), resulting from massive lysis of malignant cells that requires early recognition and management. 70 Thus, it 
Annals of International Medical and Dental Research E-ISSN: 2395-2822 | P-ISSN: 2395-2814

Vol-8, Issue-2 | March-April 2022

DOI: 10.53339/aimdr.2022.8.2.28

Page no- 219-232 | Section- Research Article (Haematology)

is important to recognize risk factors and to initiate preventive strategies. 111,12$]$ Therefore, this study was conducted with an aim to evaluate the prevalence and risk factors of developing TLS in patients with ALL. Total 50 patients admitted with ALL were included in this study after careful history taking, examination and appropriate investigations fulfilling inclusion and exclusion criteria (relapsed and secondary case of ALL, known case of CKD, epilepsy and other convulsive disorder). In this study, prevalence of TLS among ALL patients was found to be $26 \%$ $(n=13)$. The reported prevalence of TLS among ALL patients varies across the world, ranging from 19 to $63.8 \% .[11,12,13,14,15]$ By using a very inclusive definition of TLS, Truong et al. observed that the prevalence of TLS in children with ALL before and within 1 week of chemotherapy initiation was 23\%. 68 The recent study from Ethiopia by Michu $\mathrm{H}$ et al has reported $29.5 \%$ of TLS in children with all types of childhood malignancies.[13] In a study by Abdul-Basit HA et al showed the frequency of TLS in $45 \%$ which is slightly higher than our findings. 77 However, high prevalence of TLS was found in the studies of Naeem et al. and Darmon et al., in which $62.6 \%$ and $63.8 \%$ of children with acute leukaemia had TLS.[14,15] Besides, in present study, out of all 13 patients with TLS, majority $(n=9,69.23 \%)$ patients had lab TLS and 4 patients $(30.77 \%)$ had clinical TLS. Besides, most of the $(n=8,61.54 \%)$ TLS patients had spontaneous onset whereas 5 patients (38.46\%) developed TLS after initiation of chemotherapy. In a study on TLS in haematological malignancies, Wasim et al. found that $60 \%$ TLS cases were of laboratory TLS and only $20 \%$ had developed TLS spontaneously. 78 In a study of Bagshi et al.,
TLS was found in 19\% (n=74) ALL patients, among them lab TLS occurred in 69 patients $(93.24 \%)$, and only 16 patients $(21.62 \%)$ had spontaneous onset. 72 However, these wide variation in range of incidence of TLS and onset of development might be due to lack of standard criteria for diagnosis of TLS, variation in prophylaxis protocol and patients' cohort, age difference and stage of disease, delayed diagnosis, delay in initiation of chemotherapy and lack of use of effective preventive therapy. Considering the biochemical abnormalities in patients with ALL, maximum patients with TLS had significantly higher number of hyperuricemia (69.23\%) and hyperkalaemia $(61.54 \%)$ and high serum creatinine level $(23.08 \%)$ compared to non-TLS patients (2.7, 27.03 and $0 \%$ respectively) within 3 days before chemotherapy. Besides, majority patients with TLS had also significantly greater number of hyperuricemia (76.92\%), hyperkalaemia $(69.23 \%)$ and hyperkalaemia (46.15\%) compared to non-TLS patients $(0,18.92$, and $0 \%$ respectively) within 7 days after initiation of chemotherapy. Like my research findings, Naeem et al. found that the most common biochemical abnormality in ALL patients with TLS was hyperuricemia (59.3\%) followed by hyperphosphatemia (53.8\%).[14] In a study by Bagshi et al., hyperphosphatemia was also the most common (95\%) abnormality and $81 \%$ had hyperphosphatemia before induction of chemotherapy.[16] Average age of all patients was 21 years ranging from 3-70 years, wherein maximum (54\%) patients were aged less than 20 years. Greater part of the study patients was male $(62 \%)$ compared to female $(38 \%)$. There was seen no significant difference in age and sex distribution between TLS and non-TLS group ( $p$ value $>0.05$ ). Like my study findings, 
Annals of International Medical and Dental Research E-ISSN: 2395-2822 | P-ISSN: 2395-2814

Vol-8, Issue-2 | March-April 2022

DOI: 10.53339/aimdr.2022.8.2.28

Page no- 219-232 | Section- Research Article (Haematology)

several other studies also found almost similar findings. $[14,16,17,18,19]$ Sultana et al found male predominance with a male female ratio of 2.3:1. 79 Naeem et al. also found that majority (57.14\%) ALL patients were male. 74 Baset et al and Saeed et al. found that male sex was not a significant risk factor for development of TLS in ALL patients 81,69, though Bagshi et al. and Truong et al. found significant relation of male sex with TLS ( P value <0.05). [16,17] However, age and gender distribution of TLS patients in literatures was not consistent due to difference in study methodologies, geographical and demographic variations. Major part of the study patients had B-cell type ALL (66\%), though significantly higher number patients with TLS had T-cell abnormality compared to non-TLS group (61.54 vs $24.32 \%$, p value $0.021)$. These findings were similar to previous studies. $[17,20]$ Truong et al. found a strong association between $\mathrm{T}$ cell immunophenotype and development of TLS (OR 8.2, 95\% CI 4-17, $\mathrm{P}$ value $<.0001)$, whereas Baset et al. observed an significant association with both $\mathrm{B}$ and $\mathrm{T}$ cell immunophenotype to develop TLS ( OR $0.1,95 \%$ CI $0.03-0.5, \mathrm{P}$ value 0.002 and OR 8, 95\% CI1.9-32.7, $\mathrm{P}$ value $<0.002$ respectively). However, Wössmann et al. found that the children with B-precursor ALL had the highest risk to develop TLS (26.4\%).[21]

All patients of this study had complained about fever (100\%) followed by progressive pallor $(98 \%)$, history of blood transfusion $(30 \%)$, bleeding manifestation (26\%) and palpitation $(2 \%)$. Besides, the most common clinical finding was bony tenderness (58\%) followed in decreasing order splenomegaly $(48 \%)$, lymphadenopathy $(46 \%)$, oral ulcer $(46 \%)$, hepatomegaly $(36 \%)$, abdomen distension $(10 \%)$, oliguria $(8 \%)$, leg oedema $(8 \%)$, ascites $(4 \%)$, basal crepitation $(4 \%)$, jaundice $(2 \%)$, haematuria $(2 \%)$, and facial oedema (2\%). There was significant preponderance of lymphadenopathy and hepato-splenomegaly in TLS patients (92.31 and $69.23 \%$ respectively) compared to non-TLS group (29.73 and $21.62 \%$ respectively, $p$ value $<0.05)$. Like my research findings, Sultana et al. (2012) also found that all patents with ALL had fever $(100 \%)$ and bleeding manifestation was present in $46 \%$ patients. In addition, they observed splenomegaly in $96.29 \%$, bony tenderness in $76.7 \%$, lymphadenopathy in $70.0 \%$ ALL patients. 79 Similar findings regarding the clinical presentations were also observed by Davidson et al. and Hagino et al. 29,81 Initial WBC count and serum LDH of all patients was $52.51 \pm 78.70 \quad \times 109 / \mathrm{L}$ and $1591.53 \pm 2195.47 \mathrm{U} / \mathrm{L}$ respectively, wherein majority patients with TLS had WBC count $\geq 50$ $x 109 / \mathrm{L}(61.54 \%)$ and serum $\mathrm{LDH} \geq 1000 \mathrm{U} / \mathrm{L}$ $(92.31 \%)$ with significant difference compared to non-TLS patients (16.22 and $21.62 \%$ respectively, $\mathrm{p}$ value $<0.05)$. These results are comparable with previous studies. $[14,16]$ Truong et al. found significant relation of high WBC $(\mathrm{OR}=2.8, \mathrm{p}=0.026)$ count and serum LDH $(\mathrm{OR}=7.6, \mathrm{p}=<0.001)$ with development of TLS among ALL patients. 68 Belay et al. and Bagshi et al. also found that high WBC count $(>50$ x109/L) and high serum LDH (>1000U/L) was significant risk factor for developing TLS. $[16,22]$ In a study of Tasmin $\mathrm{R}$ et al., found that TLS developed in $26 \%$ with WBC count $<1$ lac and increasing up-to $100 \%$ with WBC count $>3$ lacs. 80 Furthermore, in my research work, multivariate logistic regression analysis to determine the independent predictors of developing TLS among ALL patients showed 
Annals of International Medical and Dental Research

E-ISSN: 2395-2822 | P-ISSN: 2395-2814

Vol-8, Issue-2 | March-April 2022

DOI: 10.53339/aimdr.2022.8.2.28

Page no- 219-232 | Section- Research Article (Haematology)

that serum $\mathrm{LDH} \geq 1000 \mathrm{U} / \mathrm{L}$ was the significant independent predictors of developing TLS (OR=13.07, 95\% CI: 1.93-101.23) after adjusting for age, gender, lymphadenopathy, hepatosplenomegaly and WBC level. Thus, future research may be focused on determining the additive value of this potential predictor of TLS.

\section{CONCLUSIONS}

In this study, it was observed that tumor lysis syndrome (TLS) was common in patients with

\section{REFERENCES}

1. Howard SC, Jones DP, Pui CH. The tumor lysis syndrome. N Engl J Med. 2011;364(19):1844-1854. doi:10.1056/NEJMra0904569

2. Hochberg J, Cairo MS. Rasburicase: future directions in tumor lysis management. Expert Opin Biol Ther. 2008;8(10):1595-604. doi: 10.1517/14712598.8.10.1595.

3. Chen RL, Chuang SS. Transient spontaneous remission after tumor lysis syndrome triggered by a severe pulmonary infection in an adolescent boy with acute lymphoblastic leukemia. J Pediatr Hematol Oncol. 2009;31(1):76-9. doi: 10.1097/MPH.0b013e31818ab30c.

4. Abu-Alfa AK, Younes A. Tumor lysis syndrome and acute kidney injury: evaluation, prevention, and management. Am J Kidney Dis. 2010;55(5 Suppl 3):S1-13; quiz S14-9. doi: 10.1053/j.ajkd.2009.10.056.

5. Riccio B, Mato A, Olson EM, Berns JS, Luger S. Spontaneous tumor lysis syndrome in acute myeloid leukemia: two cases and a review of the literature. Cancer Biol Ther. 2006;5(12):1614-7. doi: 10.4161/cbt.5.12.3610. Epub 2006 Dec 21.

6. Goldman SC, Holcenberg JS, Finklestein JZ, Hutchinson R, Kreissman S, Johnson FL, et al. A randomized comparison between rasburicase and allopurinol in children with lymphoma or leukemia at high risk for tumor lysis. Blood. 2001;97(10):29983003. doi: 10.1182/blood.v97.10.2998. acute lymphocytic leucaemia (ALL), wherein spontaneous onset and lab TLS was more frequent than therapy induced TLS and clinical TLS. Therefore, more vigilant assessment and monitoring to recognize and treat those patients who are at risk of TLS are essential. My study's strength was that it has focused a single and most common childhood malignancy compared to inclusion of multiple hematological malignancies in most studies. However, a large multicenter study is needed to corroborate these findings.

7. Coiffier B, Riouffol C. Management of tumor lysis syndrome in adults. Expert Rev Anticancer Ther. 2007;7(2):233-9. doi: 10.1586/14737140.7.2.233.

8. Ansari MA. Tumour lysis syndrome in haematological malignancies. JLUMHS. 2012;11(2):1-84.

9. LaRosa C, McMullen L, Bakdash S, Ellis D, Krishnamurti L, Wu HY, et al. Acute renal failure from xanthine nephropathy during management of acute leukemia. Pediatr Nephrol. 2007;22(1):132-5. doi: 10.1007/s00467-006-0287-z.

10. Beshensky AM, Wesson JA, Worcester EM, Sorokina EJ, Snyder CJ, Kleinman JG. Effects of urinary macromolecules on hydroxyapatite crystal formation. J Am Soc Nephrol. 2001;12(10):2108-2116. doi: 10.1681/ASN.V12102108.

11. Mirrakhimov AE, Voore P, Khan M, Ali AM. Tumor lysis syndrome: A clinical review. World J Crit Care Med. 2015;4(2):130-138. doi:10.5492/wjccm.v4.i2.130

12. Rajendran A, Bansal D, Marwaha RK, Singhi SC. Tumor lysis syndrome. Indian $\mathrm{J}$ Pediatr. 2013;80(1):50-4. doi: 10.1007/s12098-012-0824-7.

13. Micho H, Mohammed Y, Hailu D, Genet S. Evaluation and characterization of tumor lysis syndrome before and after chemotherapy among pediatric oncology patients in Tikur Anbessa specialized hospital, Addis Ababa, Ethiopia. BMC Hematol. 2018;18:22. doi: 10.1186/s12878-018-0117-0.

14. Naeem B, Moorani KN, Anjum M, Imam U. Tumor lysis syndrome in pediatric acute lymphoblastic 
Annals of International Medical and Dental Research

E-ISSN: 2395-2822 | P-ISSN: 2395-2814

Vol-8, Issue-2 | March-April 2022

DOI: 10.53339/aimdr.2022.8.2.28

Page no- 219-232 | Section- Research Article (Haematology)

leukemia at tertiary care center. Pak J Med Sci. 2019;35(4):899-904. doi: 10.12669/pjms.35.4.715.

15. Darmon M, Vincent F, Camous L, Canet E, Bonmati C, Braun T, et al; Groupe de Recherche en Réanimation Respiratoire et Onco-Hématologique (GRRR-OH). Tumour lysis syndrome and acute kidney injury in high-risk haematology patients in the rasburicase era. A prospective multicentre study from the Groupe de Recherche en Réanimation Respiratoire et Onco-Hématologique. Br J Haematol. 2013;162(4):489-97. doi: 10.1111/bjh.12415.

16. Al Bagshi M, Hassan ES, Sadek AO, Abbas AA. Tumor lysis syndrome in children with acute leukemia: Incidence and outcome. J Appl Hematol. 2013;4(3):1-100.

17. Truong $\mathrm{TH}$, Beyene J, Hitzler J, Abla $\mathrm{O}$, Maloney AM, Weitzman S, Sung L. Features at presentation predict children with acute lymphoblastic leukemia at low risk for tumor lysis syndrome. Cancer. 2007;110(8):1832-9. doi: 10.1002/cncr.22990.

18. Tasmeen R, Islam A, Alam ST, Begum M. Tumor Lysis Syndrome and Hyperleukocytosis in Childhood Acute Lymphoblastic Leukemia in a Tertiary Care Hospital. Mymensingh Med J. 2017;26(4):906-912.
19. Saeed F, Ali MS, Ashraf MS, Vadsaria K, Siddiqui DE. Tumour Lysis Syndrome in children with haematological cancers: Experience at a tertiary care hospital in Karachi. J Pak Med Assoc. 2018;68(11):1625-1630.

20. Wasim F, Khaskheli AK, Siddiqui AA, Tariq O. Ansari MA. Tumour Lysis Syndrome in Haematological Malignancies. J Liaquat Uni Med Heal Sci.2012;1(2):2012.

21. Wössmann W, Schrappe M, Meyer U, Zimmermann $\mathrm{M}$, Reiter A. Incidence of tumor lysis syndrome in children with advanced stage Burkitt's lymphoma/leukemia before and after introduction of prophylactic use of urate oxidase. Ann Hematol. 2003;82(3):160-5. doi: 10.1007/s00277-003-0608-2.

22. Belay Y, Yirdaw K, Enawgaw B. Tumor Lysis Syndrome in Patients with Hematological Malignancies. J Oncol. 2017;2017:9684909.

23. Cairo MS, Bishop M. Tumour lysis syndrome: new therapeutic strategies and classification. $\mathrm{Br} \mathrm{J}$ Haematol. 2004;127(1):3-11. doi: 10.1111/j.13652141.2004.05094.x.

Source of Support: Nil, Conflict of Interest: None declared 